\title{
Aplicación del Método del Análisis de Concepto al Desarrollo de la Sensibilidad Teórica Aplicada a Indagaciones Cualitativas de Carácter Inductivo: A propósito del Uso del Término "Cosmovisión"
}

\author{
Olga Rodrigo-Pedrosa ${ }^{1}$ y Jordi Caïs Fontanella ${ }^{2}$ \\ ${ }^{1}$ Escuela Superior de Enfermería del Mar - Campus de Ciencias de Salud - Universidad \\ Pompeu Fabra. España | olgarodrigopedrosa@gmail.com | https://orcid.org/0000-0001- \\ 9918-234X \\ 2 Departamento de Sociología - Facultad de Ciencias Económicas y Empresariales, \\ Departamento de sociología y Análisis de las Organizaciones - Universidad de Barcelona, \\ España | jcais@ub.edu | https://orcid.org/0000-0003-4930-303X
}

\begin{abstract}
Resumen: Introducción: La indagación cualitativa precisa de la sensibilidad teórica necesaria para poder analizar críticamente la realidad garantizando así la obtención de constructos teóricos capaces de explicar lo que ocurre con el fenómeno de estudio. Objetivo: Dar a conocer el Análisis de Concepto como un método que puede ser de utilidad para desarrollar Sensibilidad Teórica a partir de la literatura. Método: El diseño utilizado fue una adaptación de la estrategia de Análisis de Concepto de Walker \& Avans (2011). Para el muestreo se reclutó información sobre el concepto cosmovisión publicada en diccionarios, libros y artículos indexados en la base de datos Scopus y JSTOR y el metabuscador Web Of Science utilizando las palabras clave "Worldview concept" OR "World View concept". E principal criterio de inclusión fue ser materiales bibliográficos que incluyeran información conceptual sobre el término cosmovisión. Resultados: La muestra final de artículos y, materiales procedentes de citas, seleccionados una vez aplicados los criterios de inclusión y exclusión, fue de 27. A partir del análisis de los diferentes materiales bibliográficos se pudo concretar el uso del concepto Cosmovisión, sus características definitorias, sus antecedentes y consecuencias y, finalmente, sus implicaciones empíricas. Conclusión: el Análisis Teórico del término Cosmovisión nos ha permitido comprobar la adecuación del concepto para enfocar el estudio de como los grupos humanos perciben y conciben el mundo.
\end{abstract}

Palabras clave: Análisis de Concepto; Cosmovisión; Investigación Cualitativa; Sensibilidad Teórica; Teoría Fundamentada.

Application of the Concept Analysis Method to the Development of Theoretical Sensitivity Applied in Qualitative Inductive Inquiries: About the Use of the Term "Cosmovision"

Abstract: Introduction: Qualitative research requires the necessary theoretical sensitivity to be able to critically analyze reality, thus ensuring than theoretical constructs capable of explaining what is happening with the phenomenon under study can be obtained. Objective: To introduce Concept Analysis as a method that can be useful to develop Theoretical Sensibility from the literature. Method: The design used was an adaptation of the Conceptual Analysis of Walker \& Avans (2011). For the sampling, information on the Worldview Concept published in dictionaries, books and articles indexed in the Scopus and JSTOR databases and the Web Of Science meta-search engine was recruited using the keywords "Worldview Concept" OR "World View Concept". Results: The final sample of articles and materials from selected citations, after applying the inclusion and exclusion criteria, was 27 . From the analysis of the different bibliographic materials, it was possible to identify the use of the concept of Cosmovision, its defining characteristics, its antecedents and consequences and, finally, its empirical implications. Conclusion: The theoretical analysis of the term worldview has allowed us to verify the adequacy of the concept to approach the study of how human groups perceive and conceive the world.

Keywords: Concept Analysis; Grounded Theory; Theoretical Sensitivity; Worldview; Qualitative Research. 


\section{Introducción}

Según Strauss \& Corbin (1997) una de las habilidades que se requiere para hacer investigación cualitativa es la capacidad de evitar sesgos, a través del análisis crítico de las situaciones y, del desarrollo del pensamiento abstracto. Formular una teoría sobre un campo social concreto, precisa de una cierta habilidad para crear y manejar conceptos, capacidad de abstracción y la sensibilidad necesaria (Glaser, 1978). Estas habilidades se pueden englobar en lo que Glaser (1978) definió como Sensibilidad Teórica.

Para Glaser (1978) los investigadores, especialmente cuando pertenecen a un solo campo, disciplina o profesión, necesitan hacer una inmersión en la literatura para poder dotar de significado a los códigos y categorías que emergen a partir de los datos. Esto fue abalado posteriormente por Strauss \& Corbin (2002) al afirmar que, una de las fuentes que pueden ayudar al desarrollo de esa sensibilidad teorética, es la revisión de la literatura. La sensibilidad teorética es lo que permite garantizar la coherencia entre los procesos de investigación en el campo y, los de la construcción teórica, asegurando así la obtención de constructos teóricos capaces de explicar lo que sucede con el fenómeno indagado (García Hernández \& Manzano Caudillo, 2010).

La revisión de la literatura, que incluya lecturas sobre teoría e investigación, es una de las maneras de desarrollar sensibilidad teórica (Glaser, 1978; Strauss \& Corbin, 1997). La familiaridad con este tipo de publicaciones va generando una rica base de información que sensibiliza al investigador sobre el fenómeno de interés (García Hernández \& Manzano Caudillo, 2010). Sin embargo, sólo en la medida que el científico social sea sensible al fenómeno que investiga, a través de la experiencia profesional, personal y el propio proceso de investigación (Strauss \& Corbin, 2002), será capaz de distinguir el tipo de literatura que puede servirle para ampliar la comprensión del objeto de estudio (García Hernández \& Manzano Caudillo, 2010). La sensibilidad teórica, a partir de la revisión de la literatura, también es de utilidad para la adquisición de una perspectiva analítica general que permita la exploración de los datos, "a través de la utilización de las comparaciones, analogías y metáforas" (Coffey \& Atkinson, 2005, p. 148).

El Análisis de Concepto es una vía de desarrollo de teoría (Wilson, 1963) que también puede ser utilizado para aclarar, reconocer o definir conceptos que describen fenómenos humanos (McEwen \& Wills, 2018). Para McEwen \& Wills (2018) es una estrategia que puede ser utilizada para la construcción de marcos conceptuales, teorías o estudios de investigación. De forma más concreta, permite la definición de los elementos conceptuales de la teoría o constructo teórico que posteriormente servirán de guía a la investigación (McEwen \& Wills, 2018). En este sentido, el proceso de aplicar conceptos prestados o compartidos, práctica habitual de muchas disciplinas, puede conllevar una alteración de su significado por lo que se hace necesario revisarlos para verificar la idoneidad de su aplicación (Hupcey et al., 1996). También existen conceptos cuyas características no han sido correctamente descritas $u$, otros, que presentan inconsistencias entre la definición y su uso en investigación (Morse et al., 1996). Finalmente, debido a que los fenómenos, las necesidades y los objetivos cambian, los conceptos deben refinarse continuamente introduciendo variaciones para lograr un significado más claro y útil (McEwen \& Wills, 2018). Por lo tanto, tal y como planean Glaser (1978) y Strauss \& Corbin (2002), el Análisis de Concepto puede ser una estrategia adecuada para conseguir la sensibilidad teórica necesaria para llevar a cabo estudios de aproximación inductiva a través de la definición del concepto que se quiere tomar como referencia.

Greco (2008, p. 105) define en el Diccionario de Sociología la Cosmovisión como "la totalidad de nuestras interpretaciones del mundo y de nuestra vida en el mundo; asimismo, la totalidad de los valores según los cuales vivimos". Por su parte Lorena $(2013$, p. 48) en el Diccionario Básico de Antropología la define como "la forma específica de percibir y concebir el mundo natural, social y espiritual". Se trata de un concepto que permite describir la forma de vida de los grupos humanos y comparar diferentes formas de vida entre sí (Redfield, 1952). Utilizándolo se puede describir el modelo de vida de una comunidad en términos de todas las costumbres e instituciones que la distinguen (Redfield, 1952). 
Se pueden buscar los valores más fundamentales y persistentes y describirlos en términos de estos mismos valores (Benedict, 1934) o, dicho de otra manera, "su idea de la buena vida" (Redfield, 1952, p. 30). La cultura y la cosmovisión son dos herramientas esenciales para enfocar el análisis de las relaciones sociales (Carroll, 2014). Por lo tanto, el Análisis del Concepto Cosmovisión puede ser utilizado como referencia para adquirir la sensibilidad teórica necesaria para llevar estudios de indagación cualitativa enfocada en el estudio interdisciplinar de lo que hacen los grupos humanos.

El objetivo de este artículo es dar a conocer el Método de Análisis de Concepto como una estrategia que puede ser de utilidad para desarrollar Sensibilidad Teórica, a partir de la revisión de la literatura, aplicada a los estudios cualitativos de naturaleza inductiva.

\subsection{Propósito del Análisis de Concepto}

Describir el concepto Cosmovisión para que pueda ser utilizado como marco conceptual de referencia para la indagación cualitativa inductiva aplicada al estudio de como perciben y conciben el mundo grupos humanos específicos.

\section{Metodología}

\subsection{Diseño}

A principios de la década de 1960, John Wilson (1963), un científico social, desarrolló un proceso para definir conceptos con el objetivo de mejorar la comunicación y comprensión de los significados de los términos en el uso científico (en McEwen \& Wills, 2018). Posteriormente, Walker \& Avant (1986) llevaron a cabo una adaptación con el fin de simplificar el método (en McEwen \& Wills, 2018).

Para McBrien (2006, p. 42) "el análisis de conceptos juega un papel integral en la aclaración de conceptos, la construcción de teoría y la inducción de la investigación”. En el caso concreto de este estudio se analizó el concepto Cosmovisión de acuerdo con los primeros seis pasos propuestos en el método de Walter \& Avant (2011) (ver Tabla 1).

Tabla 1. Adaptación Fases Análisis de Concepto Walter \& Avant (2011)

\begin{tabular}{ll}
\hline Paso & Descripción \\
\hline Paso 1 & Seleccionar un concepto \\
Paso 2 & Determinar los objetivos o propósitos del análisis \\
Paso 3 & Identificar todos los usos del concepto que se puedan descubrir \\
Paso 4 & Determinar los atributos definitorios \\
Paso 5 & Identificar antecedentes y consecuencias \\
Paso 6 & Definir referentes empíricos \\
\hline
\end{tabular}

\subsection{Muestreo}

La recolección de datos se llevó a cabo mediante el uso de los siguientes recursos:

- Recuperación de artículos en las bases de datos, SCOPUS y JSTOR y el metabuscador WEB OF SCIENCE

- Consulta de libros específicos identificados a partir de las citas de los artículos recuperados 
La estrategia de búsqueda utilizada en todos los casos fue ("World View Concept" OR "Worldview Concept"). En cuanto a los criterios de inclusión se reclutaron todos los artículos que incluyeran "Usos del Concepto" "Atributos", "Antecedentes", "Consecuencias", "Referentes Empíricos" en lengua "española, "inglesa", "francesa", "portuguesa" e "italiana" sin límite de tiempo. Se excluyeron aquellos artículos que utilizaran de forma aplicada el concepto Cosmovisión, para describir un fenómeno concreto $\mathrm{o}$, la literatura gris procedente de comunicaciones, jornadas, tesis doctorales, revisiones de libros, etc.

Tal y como se puede ver en la Figura 1 la muestra final estuvo compuesta por 13 artículos y 14 libros específicos identificados a partir de búsqueda de citas.

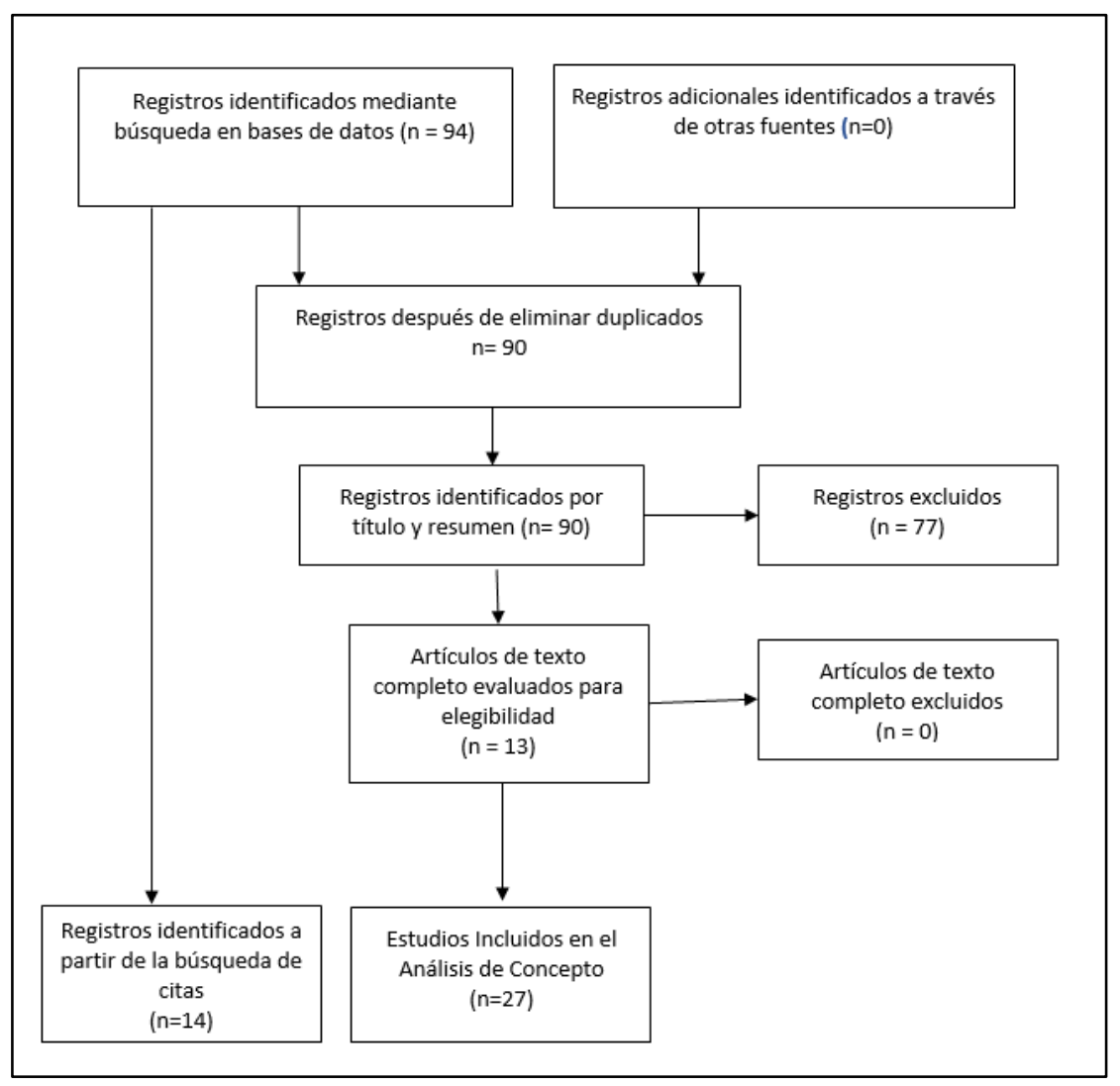

Fig. 1. Descripción de la búsqueda para la definición del concepto Cosmovisión. Nota: Elaboración Propia

\subsection{Análisis de Datos}

Para el proceso de análisis del contenido del texto completo de los documentos seleccionados a partir de la búsqueda bibliográfica (ver Figura 1) se tomaron como referencia las fases del análisis del concepto propuestas por Walter \& Avant (2011) (ver pasos 3, 4, 5 y 6 en Tabla 1 ).

En una primera fase, los datos procedentes de los 13 artículos y 14 registros identificados a partir de la búsqueda de citas se codificaron y agruparon en las categorías "Uso del concepto", "Atributos Definitorios", "Antecedentes y Consecuencias" y "Referentes Empíricos". A continuación, se elaboró una matriz para cada categoría que facilitó la visualización de la información. En una tercera fase se enfocó la información de las matrices para identificar aquellos elementos susceptibles de ser utilizados como fuente de Sensibilidad Teórica.

Finalmente, para asegurar la auditabilidad se utilizó el programa MAXQDA2020 como soporte operativo durante todo del proceso. 


\section{Resultados}

\subsection{Uso del Concepto}

La Cosmovisión es definida como "la perspectiva del universo que es característica de un pueblo" (Redfield, 1952, p.30). Es la "forma en que un grupo humano le da sentido a su entorno, a la vida y al universo" (Ani, 1980, p. 4). Se trata de un grupo de conceptos que "permiten tanto describir una forma de vida como comparar diferentes formas de vida entre sí" (Redfield, 1952, p. 30). Según Kearney (1984, p. 1) es un "macro pensamiento culturalmente organizado" que para Cobern (1996) incluye las presuposiciones de un pueblo que determinan en parte su comportamiento y toma de decisiones. Es la idea que las personas tienen sobre lo que es bueno y malo, correcto e incorrecto, justo o injusto (Goldstein \& Keohane, 1993; Rowland \& Theye, 2008).

Para Ferrer-Santos (1981) el término Cosmovisión hace referencia a una visión de conjunto relativa al mundo en el que actúa el hombre que es una síntesis conceptual de la experiencia, impregnada del tono característico que le da el individuo o una época histórica concreta. Jones (1992) completa la definición afirmando que, es precisamente esa experiencia vivida, la que constituye la mirada a través de la cual el mundo, percibido por los sentidos humanos, se reduce a un hecho susceptible de ser descrito

Las cosmovisiones son relevantes como capacidad humana para crear orden, significado y valor en un mundo que puede mostrarse caótico (Sheikh, 2018). Se trata de un concepto que puede ser utilizado para hacer referencia a filosofías de vida, ideologías holísticas y perspectivas tanto culturales como religiosas (Sheikh, 2018). Finalmente, para Walsh \& Middleton (1984, p. 35) el constructo Cosmovisión permite dar respuesta a las cuestiones "¿Quiénes somos nosotros? ¿Dónde estamos nosotros? ¿Por qué sufrimos? y ¿Cuál es el remedio?". Por lo tanto, permite indagar en los problemas identificados por los humanos que integran los grupos humanos y, la solución que plantean para aliviarlos (Jensen, 1997).

\subsection{Atributos Definitorios}

El paso 4 del método de Walker \& Avant's (2011) es determinar los atributos definitorios del concepto. En el caso del concepto Cosmovisión estos son:

- Todas las cosmovisones presentan una misma estructura universal (Dilthey, 1954) determinada por la regularidad psíquica que es común a los seres humanos y, a partir de la cual, deciden cuestiones acerca de la significación y el sentido del mundo (Dilthey, 1954). Se trata de una estructura compuesta por siete categorías universales y relacionadas entre sí (Cobern, 1996, p. 584): "1) el Yo; 2) el No-Yo; 3) la Clasificación; 4) la Relación; 5) la Causalidad; 6) el Tiempo y 7) el Espacio". Cada una de ellas se compone a su vez de presuposiciones lógicamente relacionadas de manera que, los grupos de personas, pueden identificarse por variaciones en el contenido de estas categorías (Cobern, 1996).

- Las ideas del mundo favorecen la comprensión de la vida y conducen a fines vitales (Dilthey, 1954).

- Las ideas del mundo van cambiando de generación en generación manteniéndose sólo aquellas que son más viables (Dilthey, 1954).

- La cosmovisión proporciona una base no racional para el pensamiento, la emoción y el comportamiento, en forma de presuposiciones sobre cómo es realmente el mundo y qué constituye un conocimiento válido e importante sobre él (Cobern, 1996)

- Las cosmovisión "no es un producto del pensamiento sino que surge de la experiencia de la vida" (Dilthey, 1954, p. 119). El origen de su formación es la experiencia entendida como la decantación resultante de actos realizados más que como tarea inicial (FerrerSantos, 1981). Por lo tanto, las presuposiciones están sujetas a los cambios derivados de la experiencia (Cobern, 1996). 
- Existen una "diversidad de cosmovisiones determinadas por el clima, las razas, las nacionalidades determinadas por la historia y la formación estatal, las limitaciones de épocas y períodos, condicionadas temporalmente y las cuales concurren entre si" (Dilthey, 1954, p. 117).

- Las diferentes cosmovisiones luchan entre sí para "dominar sobre las almas" (Dilthey, 1944, p. 117).

\subsection{Antecedentes y Consecuencias}

El siguiente paso en el análisis de concepto es identificar sus antecedentes y consecuencias. Los antecedentes son los incidentes o eventos que sucedieron antes de la ocurrencia del concepto (Walker \& Avant, 2011).

En cuanto a los antecedentes según Naugle (2002) Kant (1724-1804) acuñó el término "Weltanschauung", que más tarde fue traducido al inglés como "Cosmovisión" o "Worldview", para referirse a la intuición que tiene el ser humano en relación con lo que es el mundo. Kant (1952, p. 111) Introdujo este concepto compuesto por Welt (mundo) y Aunschaauung (visión o perspectiva), para describir "la capacidad del ser humano para ordenar un mundo lleno de infinitas posibilidades de ser percibido". En palabras de Hedlund-De Witt (2013) para Kant es el intento de la razón humana para formarse una visión comprensiva que permite articular la relación entre el sujeto humano y los objetos que le rodean, en el marco de una comprensión denominada "weltanschauung".

Pese a que el vocablo procede de Kant (en Naugle, 2002), para Torralba (2010) uno de los máximos teóricos de su utilización fue el filósofo Dilthey. Para Dilthey (1954, p. 41) el término Cosmovisión hace referencia a que es la propia vida la que crea, a partir de cada individuo, su propio mundo y que, para Redfield (1952), atiende especialmente a la forma en que un hombre, en una sociedad particular, se hace a sí mismo en relación con todo lo demás.

\subsection{Referentes Empíricos}

El paso final del análisis del concepto es definir los referentes empíricos definidos como las categorías o clases para medir el concepto. Para muchos conceptos, como es el caso del concepto Cosmovisión, los atributos definitorios y los referentes empíricos son idénticos (Walker \& Avant, 2011). A partir de la revisión de la literatura no se encontró ningún instrumento empírico para medir la Cosmovisión. Sin embargo, sí que se han encontrado referentes empíricos en cuanto a ámbitos de investigación donde se ha aplicado previamente el concepto Cosmovisión.

La comprensión filosófica de la cosmovisión de Kant ha sido aplicada en un sentido empírico por psicólogos contemporáneos traduciéndola a esquemas cognitivos reduciendo así la complejidad de la experiencia (Beck, 1979; Janoff-Bulman, 1989). Des de esta perspectiva, las visiones del mundo se adaptan, dentro de un marco cognitivo, que se interesa principalmente en como las personas desarrollan visiones del mundo compartidas como estructura cognitiva, que aplican para dar sentido y llegar así a un acuerdo con el mundo (Johnson et al., 2011; Kearney, 1984; Koltko-Rivera, 2004).

Por su parte, Weber (2000) demostró el valor de aplicar el análisis de la Cosmovisión en las ciencias sociales. Ese enfoque está más interesado en las sociopolíticas a desarrollar y compartir supuestos sobre la realidad social (Johnson et al., 2011; Kearney, 1984; Koltko-Rivera, 2004; Rowland \& Theye, 2008). Bajo esta perspectiva el análisis de la Cosmovisión puede ser utilizado para estudiar la formación de actitudes colectivas y la forma en que las diferentes comunidades se relacionan entre sí (Goplen \& Plant, 2015; Greenberg et al., 1997; Major et al., 2007). 
Finalmente las cosmovisiones son relevantes como narrativas que explican el lugar de una persona en el mundo, identifican adversarios y vinculan a la persona con un propósito mundano y trascendente (Edwards, 2014; Roislien, 2007).

\section{Discusión}

Tal y como han evidenciado los "Referentes Empíricos" del concepto cosmovisión puede ser un marco conceptual adecuado para enfocar como perciben y conciben el mundo grupos humanos específicos (Beck, 1979; Goplen \& Plant, 2015; Greenberg et al., 1997; Janoff-Bulman, 1989; Jensen, 1997; Johnson et al., 2011; Kearney, 1984; Major et al., 2007; Redfield, 1952; Rowland \& Theye, 2008; Sheikh, 2018; Weber, 2000).

A partir del análisis del "Uso del Concepto", se ha podido identificar que las técnicas de generación de datos más coherentes, para la indagación de las cosmovisiones, podrían ser aquellas que permiten situar la experiencia en el contexto sociohistórico ya que, según Dilthey (1954), Ferrer-Santos (1981), Jones (1992) y Cobern (1996), son elementos que influyen en su construcción. En este sentido, la propuesta de entrevista fenomenológica de Seidman (2013) podría ser una herramienta muy adecuada para este tipo de indagaciones ya que, propone explorar en el significado, situando previamente la experiencia en el contexto sociohistórico donde se produce.

Finalmente, a través del establecimiento de los "Atributos Definitorios" del concepto, se han identificado elementos que pueden ser utilizados como referencia para iniciar el proceso de análisis basado en la Teoría Fundamentada (Glaser \& Strauss, 2002) al facilitar el llevar a cabo comparaciones con los datos (Coffey \& Atkinson, 2005, p. 148). Concretamente, la aplicación a los datos durante la fase de "codificación abierta" como "herramienta analítica" (Strauss \& Corbin, 2002, p. 98) de las preguntas "¿Quiénes somos nosotros? ¿Dónde estamos nosotros? ¿Por qué sufrimos? y ¿Cuál es el remedio" (Walsh \& Middleton, 1984) puede ayudar a "generar ideas o maneras de mirar a los datos" (Strauss \& Corbin, 2002, p. 98) con el objetivo de "aumentar la sensibilidad, ayudar al usuario a reconocer los sesgos y superar el bloqueo analítico" (Strauss \& Corbin, 2002, p. 96). Además los elementos "El yo", "El No-Yo", "la Clasificación", "La relación", "La causalidad", "el Tiempo", "El espacio" (Cobern, 1996; Dilthey, 1954), "lo bueno y malo", " lo correcto e incorrecto" y "lo justo e injusto" (Goldstein \& Keohane, 1993; Rowland \& Theye, 2008) pueden facilitar el enfoque inicial de categorías durante la codificación axial (Strauss \& Corbin, 2002).

La principal limitación de este estudio tiene que ver con que para analizar el concepto cosmovisión se ha utilizado como referencia información procedente de bases de datos propias del contexto occidental. Esto puede conllevar un uso desde una perspectiva teórica excesivamente colonial y eurocéntrica. Por ello, a priori, esta sensibilidad teorética sólo sería de utilidad para aplicarla en la indagación de los grupos humanos específicos de este contexto y habría que indagar su pertinencia para otros.

\section{Conclusión}

El Método de Análisis de Walter \& Avant (2011) parece ser adecuado para el desarrollo de sensibilidad teorética a partir del concepto Cosmovisión. En general, se ha podido evidenciar la adecuación del concepto para el enfoque de como perciben y conciben el mundo los grupos humanos. De forma más específica, también ha orientado la selección de la técnica de recogida de datos y, sobre todo, el enfoque inicial del análisis de los datos utilizando como referencia la Teoría Fundamentada. Llegado a este punto sólo quedaría aplicar el enfoque en el estudio de un grupo humano para ver cómo se comporta a nivel práctico. 


\section{Referencias}

Ani, M. (1980). Let the Circle Be Unbroken. New York: Nkonimfo.

Beck, A. T. (1979). Cognitive Therapy of Depression (The Gilford Clinical Psychology and Psychology Series). New York: Guilford.

Benedict, R. (1934). Patters of culture. Boston \& New York: A Mariner Book.

Carroll, K. (2014). An Introduction to African-Centered Sociology: Worldview, Epistemology, and Social Theory. Critical Sociology, 40(2), 257-270. doi.org/10.1177/0896920512452022

Cobern, W. W. (1996). Worldview theory and conceptual change in science education. Scientific Literacy and Cultural Studies project. Science Education, 80(5), 1-31.

Coffey, A., \& Atkinson, P. (2005). Encontrar sentido a los datos cualitativos. Estrategias complementarias de investigación. Alicante: Servicio de Publicaciones de la Universidad de Alicante.

Dilthey, W. (1954). Teoría de las concepciones del Mundo. Madrid: Revista de Occidente S.A.

Edwards, J. A. (2014). The Mythology of Suffering and Redemption in the Discourse of al Qaeda. In D. Whitt and J.R. Perlich (Ed.), Myth in the Modern World: Essays on Intersections with Ideologiy and Culture (pp. 83-1010). Jefferson, NC: McFarlant.

Ferrer-Santos, U. (1981). Filosofía y cosmovisión. Anuario Filosófico, 14(2), 173-182.

García Hernández, G., \& Manzano Caudillo, J. (2010). Procedimientos metodológicos básicos y habilidades del investigador en el contexto de la teoría fundamentada. Iztapalapa, Revista de Ciencias Sociales y Humanidades, 69, 17-39.

Glaser, B. (1978). Theoretical Sensivility: advances in the methodology of grounded theory. Mill Valley, CA: The Sociology Press.

Goldstein, J., \& Keohane, O. (1993). Ideas and Foreign Policy: An Analytical Framework. In J. Goldstein \& R. O. Keohane (Eds.), Ideas and Foreign Policy: Beliefs, Institutions, and Political Chance (pp. 3-30). New York: Cornell University Press.

Goplen, J., \& Plant, A. (2015). A religious Worldview Protecting One's Meaning System Through Religious Prejudice. Personality \& Social Psychology Bulletin, 41, 1474-1487. doi:10.1177/0146167215599761.

Greco, O. (2008). Cosmovisión. Diccionario de Sociologia. Buenos Aires: Valletta Ediciones

Greenberg, J., Solomon, S., \& Pyszcznski, T. (1997). Terror Management Theory of Self-Esteen and Cultural Worldviews: Empirical Assessments and Conceptual Refinements. Advances in Experimental Social Psychology, 29, 61-139. doi: 10.1016/S0065-2601(08)60016-7.

Hupcey, J., Morse, J., Lenz, E., \& Tasón, M. (1996). Wilsonian Methods of Concept Analysis: A Critique. Scholarly Inquiry for Nursing Practice, 10(3), 185-210. doi: 10.1891/08897182.10.3.185

Janoff-Bulman, R. (1989). Assumptive Worlds and the Stress of Traumatic Events: Applications of the Schema Construct. Social Cognition, 7, 113-136. doi: 10.1521/soco. 1989.7.2.113

Jensen, L. A. (1997). Different worldviews, different morals: America's culture war divide? Human Development, 40(6), 325-344. doi: 10.1159/000278737

Johnson, K. A., Hill, E. D., \& Cohen, A. B. (2011). Integrating the Study of Culture and Religion: Toward a Psychology of Worldview. Social and Personality Psychology Compass, 5, 137152. doi: 10.1111/j.1751-9004.2010.00339

Jones, M. (1992). Political science and the black political experience: issues in epistemology and relevance. National Political Science Review, 3, 25-39. doi: 10.4324/9781215080420-20

Kant, I. (1952). The Critique of Judgment. Oxford, UK: Clarendon.

Kearney, M. (1984). World view. Novato, CA: Chandler \& Sharp Publishers, Inc.

Koltko-Rivera, M. E. (2004). The Psychology of Worldviews. Review of General Psychology, 8, 358. doi: 10.1037/1089-2680.8.1.3

Lorena, A. (2008). Cosmovisión. Diccionario Básico de Antropologia. Quito, Ecuador: Abya-Yala 
Major, B., Kaiser, R., O'Brien, L. T., \& McCoy, S. K. (2007). Perceived discrimination as worldview threat or worldview confirmation: Implications for self-esteem. Journal of Personality and Social Psychology, 92 (6), 1068-1089. doi: 10.1037/0022-3514.92.6.1068

McBrien, B. (2006). A concept analysis of spirituality. British Journal of Nursing, 15(1), 42-45. doi: 10.12968/bjon.2006.15.1.20309

McEwen, M., \& Wills, E. (2018). Concept Development: Clarifying Meaning of Terms. In M. McEwen \& E. Wills, Theoretical Basis for Nursing ( $5^{\circ}$ edition, pp. 72-91). Houston, Texas: Wolters Kluwer.

Mead, G. H. (1934). Mind, Self \& Society. London, UK: The University of Chicago Press.

Morse, J., Hupcey, J., Mitcham, C., \& Lenz, E. (1996). Concept Analysis in Nursing Research: A Critical Appraisal. Scholarly Inquiry for Nursing Practice, 10(3), 253-277. doi: 10.1891/08897182.10.3.253

Naugle, D. (2002). Worldview: The History of Concept. Cambridge, UK: William B. Eerdmans Publishing Company.

Redfield, R. (1952). The Primitive Word View. Proceedings of the American Philosophical Society, 96(1), 30-36.

Roislien, H. E. (2007). Living with Contradiction: Examining the Worldview of the Jewish Settlers in Hebron. International Journal of Conflict and Violence, 1, 169-184. doi: 10.4119/UNIBI/ijcv.14.

Rowland, R., \& Theye, K. (2008). The Symbolic DNA of Terrorism. Communication Monographs, 75, 52-85. doi: 10.1080/03637750701885423

Seidman, I. (2013). Interviewing as qualitative research. A guide for researchers in education and the social sciences. London: Teachers College Press

Sheikh, M. K. (2018). Worldview analysis. The Oxford Handbook of Global Studies, March, 157172. doi: $10.1093 /$ oxfordhb/9780190630577.013.13

Strauss, A., \& Corbin, J. (1997). Grounded Theory in Practice. Thousand Oaks, California: SAGE.

Strauss, A. L., \& Corbin, J. (2002). Bases de la investigación cualitativa: técnicas y procedimientos para desarrollar la teoría fundamentada. Medellin: Universidad de Antioquia.

Torralba, F. (2010). La creación de una cosmovisión frente a la dispersión y sobresaturación. Ars Brevis, 16, 231-247

Walker, L. O., \& Avant, K. C. (2011). Strategies for Theory Construction in Nursing (5th ed.). Norwalk, CT: Appleton \& Lange.

Walsh, B., \& Middleton, J. R. (1984). The Transforming Vision. Downers Grove, Illinois: IVP Academic.

Weber, M. (2000). The Protestant Ethic and the 'Spirit' of capitalism and Other Writings. New York: Penguin/Putnam.

Wilson, J. (1963). Thinking with concepts. Cambrigge: Cambridge University Press. 